\title{
Comparative speed of kill after treatment with Simparica (sarolaner) and Advantix (imidacloprid + permethrin) against induced infestations of Dermacentor reticulatus on dogs
}

\author{
Csilla Becskei ${ }^{1}$, Thomas Geurden ${ }^{1 *}$, Heidi Erasmus², Otto Cuppens' ${ }^{1}$, Sean P. Mahabir ${ }^{3}$ and Robert H. Six ${ }^{3}$
}

\begin{abstract}
Background: Ticks are common ectoparasites that infest dogs globally. Acaricides with rapid and sustained speed of kill are critical to control infestations and to reduce the risk of disease transmission. This study evaluated the speed of kill for 5 weeks after a single dose of orally administered Simparica (sarolaner) against induced infestations with Dermacentor reticulatus on dogs, compared to Advantix Spot-on solution for dogs (imidacloprid + permethrin).

Methods: Twenty four dogs were randomly allocated to treatment with either a placebo tablet, a sarolaner tablet (at 2 to $4 \mathrm{mg} / \mathrm{kg}$ ) or with Advantix as per label instructions. Dogs were treated on Day 0 and tick counts were performed in situ at 8 and 12 hours and with removal of the ticks at 24 hours after treatment and subsequent re-infestations on Days 7, 14, 21, 28 and 35. Acaricidal efficacy was determined at each time point relative to live tick counts from the placebo-treated dogs.

Results: Based on arithmetic (geometric) mean tick counts, the efficacy of sarolaner was $\geq 75.6 \%$ (89.6 \%) within 8 hours of treatment and tick counts were significantly lower than placebo and imidacloprid + permethrin-treated dogs $(P<0.0001)$, while imidacloprid + permethrin had no significant reduction $(P \geq 0.3990)$ at 8 or 12 hours after treatment. Sarolaner killed all ticks on the dogs within 24 hours after treatment, while imidacloprid + permethrin efficacy was only $48.1 \%$. After weekly re-infestations sarolaner significantly reduced the tick counts versus placebo within 8 hours on Days 7, 14 and $35(P \leq 0.0239)$, and at 12 hours and 24 hours $(P \leq 0.0079)$ until Day 35.Sarolaner efficacy was $\geq 95.8 \%$ within 24 hours for 35 days. Significantly more live ticks $(P \leq 0.0451)$ were recovered from imidacloprid + permethrin-treated dogs than from sarolaner-treated dogs at 24 hours after infestation on all days. There were no sarolaner-related adverse reactions during the study.

Conclusions: This study demonstrated that Simparica ${ }^{\text {TM }}$ had a faster and more consistent speed of kill against $D$. reticulatus compared to Advantix . The rapid and consistent efficacy within 24 hours for 5 weeks after a single oral dose of Simparica ${ }^{\text {tm }}$ provides effective and reliable control of D. reticulatus and reduces the risk of transmission of tick-borne diseases.
\end{abstract}

Keywords: Dermacentor reticulatus, Sarolaner, Simparica" ${ }^{\text {mM }}$, Imidacloprid + permethrin, Speed of kill, Tick, Dog, Oral, Isoxazoline, Advantix

\footnotetext{
* Correspondence: thomas.geurden@zoetis.com

'Zoetis, Veterinary Medicine Research and Development, Mercuriusstraat 20,

Zaventem B-1930, Belgium

Full list of author information is available at the end of the article
} 


\section{Background}

Dermacentor reticulatus, also known as the ornate dog tick or meadow tick, infests dogs throughout Europe. Recently there have been several reports about the potential expansion and increased prevalence of this tick in Europe [1-7]. D. reticulatus is also a known vector for Babesia canis, an intracellular protozoan parasite that is a threat to dogs as a cause of anemia, thrombocytopenia, and various clinical signs, ranging from mild, nonspecific illness to peracute collapse and death $[8,9]$. As awareness of tick-borne diseases has increased, tick control and prevention have taken on a new importance. Until recently, topically administered parasiticides with contact activity have been the only available option for tick control on dogs. These products are generally perceived to have the potential to kill or repel ticks before they bite, reducing the risk of disease transmission.

Simparica" (sarolaner) is a novel parasiticide from the isoxazoline class of molecules, and provides a new effective alternative for the control of ticks, including $D$. reticulatus for at least 5 weeks after a single oral dose [10]. As systemically active compounds require the tick to bite to receive a lethal dose of the parasiticide, it is important that these compounds act rapidly and consistently. A single dose of sarolaner provides greater than $90 \%$ efficacy within 24 hours for at least 4 weeks against common ticks worldwide and within 12 hours against Ixodes ricinus, the most widely distributed tick species in Europe [11]. While tick efficacy claims are based on evaluation at 48 hours after treatment or re-infestation [12], the speed of acaricidal activity is critical in preventing feeding and thus reducing the risk of disease transmission which occurs after the infected tick is attached and feeding for at least 24 to 48 hours [13, 14]. The present study was conducted to evaluate and compare the speed of kill of a single oral dose of sarolaner with that of imidacloprid + permethrin (Advantix Spot-on solution for dogs), against an existing infestation and against weekly re-infestations with $D$. reticulatus for a period of 5 weeks after treatment.

\section{Methods}

\section{Ethical approval}

Study procedures were in accordance with the World Association for the Advancement of Veterinary Parasitology (WAAVP) guidelines for evaluating the efficacy of parasiticides for the treatment, prevention and control of flea and tick infestation on dogs and cats [12]. The protocol was reviewed and approved by the Zoetis Ethical Review Board and the local Animal Welfare Committee. Masking of the study was assured through the separation of study functions. All personnel conducting observations or animal care or performing infestations and counts were masked to treatment allocation.

\section{Animals}

Twenty-four (8 male and 16 female) purpose-bred Beagle and mixed breed dogs from 9 months to 6 years of age and weighing from 10.8 to $24.8 \mathrm{~kg}$ were used in the study. Each dog had undergone an adequate wash-out period to ensure that no residual ectoparasiticide efficacy remained from any previous administration. Dogs were individually housed and were acclimatized to these conditions for at least 8 days prior to treatment. Dogs were fed an appropriate maintenance ration of a commercial canine diet for the duration of the study. Water was available ad libitum. All dogs were given a physical examination to ensure that they were in good health at enrollment and were suitable for inclusion in the study. General health observations were performed twice daily throughout the study.

\section{Design}

The study followed a randomized complete block design. Dogs were ranked according to decreasing tick counts into blocks of three animals, and within each block a dog was randomly allocated to one of three treatment groups. There were eight dogs per treatment group. The average hair length in each group was similar.

\section{Treatment}

On Day 0, dogs received either a placebo tablet, an appropriate Simparica chewable tablet (sarolaner at 2 to $4 \mathrm{mg} / \mathrm{kg}$ ) or a topical application of Advantix Spot-on solution for dogs (imidacloprid + permethrin at 10 to $25 \mathrm{mg} / \mathrm{kg}$ imidacloprid and 50 to $125 \mathrm{mg} / \mathrm{kg}$ permethrin). The Day -2 bodyweights were used to calculate the appropriate dosage to be administered. The tablet(s) were administered by hand pilling to ensure accurate and complete dosing. Each dog was observed for several minutes after dosing for evidence that the dose was swallowed, and for potential adverse events associated with treatment administration. Dogs were observed approximately two hours after dosing for evidence of emesis. The imidacloprid + permethrin treatment was applied topically following the label directions.

\section{Tick infestation and assessment}

The $D$. reticulatus strain used for infestation was established in 2007 in Ireland. The colony was genetically enriched by the addition of wild-caught ticks from the Netherlands in 2009 and 2012. Tick infestations were performed on Days -7,-2, 7, 14, 21, 28 and 35. Prior to each infestation, the dog was sedated to enhance tick attachment, and $50( \pm 5)$ viable unfed adult $D$. reticulatus (1:1 male:female) was directly applied to each animal. Each dog was examined to remove and count live ticks at 48 hours after the infestation on Day -7 , to confirm host suitability and counts were used for allocation to 
treatments. On Days 0, 7, 14, 21, 28 and 35, the dogs were examined 8 and $12( \pm 0.5)$ hours after treatment or each subsequent weekly re-infestation, and live ticks were counted in situ; the dogs were systematically examined so that the entire body surface was carefully examined once. At $24( \pm 0.5)$ hours after treatment and each subsequent weekly re-infestation, the dogs were examined and then thoroughly combed to count and remove ticks. Each dog was examined for at least 10 minutes. If ticks were encountered in the last minute, combing was continued in one minute increments until no ticks were encountered.

\section{Statistical analysis}

The individual dog was the experimental unit. Data for post-treatment live (free plus attached) tick counts were summarized with arithmetic (AM) and geometric (GM) means by treatment group and time-point. Tick counts were transformed by the $\log _{\mathrm{e}}($ count +1$)$ transformation prior to analysis in order to stabilize the variance and normalize the data. Using the PROC MIXED procedure (SAS 9.2, Cary NC), transformed counts were analyzed using a mixed linear model for repeated measures. The fixed effects were treatment, time-point and the treatment by time-point interaction. Random effects included room, block within room, block by treatment interaction within room, and error. Testing was two-sided at the significance level $\alpha=0.05$.
The assessment of acaricidal efficacy was based on the percent reduction in the arithmetic and geometric mean live tick counts relative to placebo, as suggested by the most recent WAAVP guidelines [12], and was calculated using Abbott's formula:

$$
\% \text { reduction }=100 \times \frac{\text { mean count }(\text { placebo })-\text { mean count }(\text { treated })}{\text { mean count }(\text { placebo })}
$$

\section{Results}

The results of the tick counts at each time-point are provided in Tables 1, 2 and 3, and in Fig. 1. Placebotreated dogs maintained adequate tick infestations throughout the study. AM (GM) live tick counts for the placebo-treated dogs at the 8-hour tick count ranged from 23.5 (21.7) to 35.8 (34.5); at the 12-hour count from 20.5 (18.3) to 33.6 (32.3); and at the 24-hour count from 25.0 (23.0) to 30.1 (28.7).

Eight hours after treatment the reduction in AM (GM) tick counts was $75.6 \%$ (89.6 \%) for sarolaner and $5.6 \%$ (14.6\%) for imidacloprid + permethrin. Geometric mean live tick counts were significantly lower $(P<0.0001)$ than placebo for sarolaner but not forimidacloprid + permethrin $(P=0.6216)$.Eight hours after the subsequent weekly reinfestations the reduction in AM (GM) tick counts for sarolaner ranged from $30.0 \%$ (29.6 \%) to $65.0 \%$ (66.4 \%). GM live tick counts for sarolaner were significantly lower $(P \leq 0.0239)$ than placebo, except on Days 21 and 28.

Table 1 The Range, Arithmetic (AM) and Geometric (GM) Mean live Dermacentor reticulatus counts, and efficacy relative to placebo at 8 hours after treatment and re-infestations for dogs treated with either a single oral dose of sarolaner or a single topical application of imidacloprid+permethrin on Day 0

\begin{tabular}{|c|c|c|c|c|c|c|c|}
\hline \multirow[t]{2}{*}{ Treatment } & & \multicolumn{6}{|c|}{ Day of treatment or re-infestation } \\
\hline & & 0 & 7 & 14 & 21 & 28 & 35 \\
\hline \multirow[t]{3}{*}{ Placebo } & Range & 23 to 43 & 12 to 34 & 26 to 43 & 13 to 40 & 21 to 39 & 20 to 43 \\
\hline & AM & 33.8 & 23.5 & 35.8 & 28.4 & 32.4 & 35.4 \\
\hline & $G M^{c}$ & $32.4^{a}$ & $21.7^{\mathrm{a}}$ & $34.5^{\mathrm{a}}$ & $26.4^{a}$ & $31.2^{\mathrm{a}}$ & $33.7^{\mathrm{a}}$ \\
\hline \multirow[t]{6}{*}{ Sarolaner } & Range & 0 to 31 & 6 to 21 & 8 to 22 & 12 to 27 & 13 to 30 & 12 to 33 \\
\hline & AM & 8.3 & 13.1 & 12.5 & 19.9 & 22.1 & 21.1 \\
\hline & AM Efficacy (\%) & 75.6 & 44.1 & 65.0 & 30.0 & 31.7 & 40.3 \\
\hline & $G M^{c}$ & $3.4^{b}$ & $12.1^{\mathrm{b}}$ & $11.6^{\mathrm{b}}$ & $18.6^{\mathrm{a}}$ & $20.6^{a}$ & $19.8^{b}$ \\
\hline & GM Efficacy (\%) & 89.6 & 44.1 & 66.4 & 29.6 & 33.9 & 41.2 \\
\hline & $P$-value vs. placebo & $<0.0001$ & 0.0157 & $<0.0001$ & 0.1349 & 0.0766 & 0.0239 \\
\hline \multirow[t]{7}{*}{ Imidacloprid+permethrin } & Range & 6 to 45 & 0 to 33 & 2 to 18 & 0 to 18 & 5 to 20 & 7 to 42 \\
\hline & AM & 31.9 & 9.9 & 8.9 & 6.9 & 14.3 & 19.5 \\
\hline & AM Efficacy (\%) & 5.6 & 58.0 & 75.2 & 75.8 & 56.0 & 44.9 \\
\hline & $G M^{c}$ & $27.6^{a}$ & $4.2^{c}$ & $7.2^{b}$ & $4.7^{b}$ & $12.8^{\mathrm{b}}$ & $16.8^{b}$ \\
\hline & GM Efficacy (\%) & 14.6 & 80.6 & 79.0 & 82.0 & 58.9 & 50.2 \\
\hline & $P$-value vs. placebo & 0.6216 & $<0.0001$ & $<0.0001$ & $<0.0001$ & 0.0074 & 0.0331 \\
\hline & P-value vs. sarolaner & $<0.0001$ & 0.0077 & 0.2152 & 0.0005 & 0.1917 & 0.6461 \\
\hline
\end{tabular}

${ }^{\mathrm{c}}$ Geometric means within columns with the same superscript are not significantly different $(P>0.05)$ 
Table 2 The Range, Arithmetic (AM) and Geometric (GM) Mean live Dermacentor reticulatus counts, and efficacy relative to placebo at 12 hours after treatment and re-infestations for dogs treated with either a single oral dose of sarolaner or a single topical application of imidacloprid+permethrin on Day 0

\begin{tabular}{|c|c|c|c|c|c|c|c|}
\hline \multirow[t]{2}{*}{ Treatment } & & \multicolumn{6}{|c|}{ Day of treatment or re-infestation } \\
\hline & & 0 & 7 & 14 & 21 & 28 & 35 \\
\hline \multirow[t]{3}{*}{ Placebo } & Range & 23 to 41 & 6 to 35 & 23 to 41 & 12 to 37 & 19 to 34 & 12 to 43 \\
\hline & AM & 33.6 & 20.5 & 31.3 & 27.0 & 28.5 & 33.3 \\
\hline & $G^{c}$ & $32.3^{\mathrm{a}}$ & $18.3^{\mathrm{a}}$ & $30.1^{\mathrm{a}}$ & $25.2^{\mathrm{a}}$ & $27.3^{\mathrm{a}}$ & $30.6^{\mathrm{a}}$ \\
\hline \multirow[t]{6}{*}{ Sarolaner } & Range & 0 to 7 & 3 to 15 & 6 to 13 & 1 to 24 & 9 to 24 & 10 to 28 \\
\hline & AM & 1.4 & 8.6 & 8.6 & 11.0 & 15.6 & 16.9 \\
\hline & AM Efficacy (\%) & 95.9 & 57.9 & 72.4 & 59.3 & 45.2 & 49.2 \\
\hline & $\mathrm{GM}^{\mathrm{c}}$ & $0.6^{\mathrm{b}}$ & $7.7^{\mathrm{b}}$ & $8.2^{\mathrm{b}}$ & $8.4^{\mathrm{b}}$ & $14.5^{\mathrm{b}}$ & $15.7^{\mathrm{b}}$ \\
\hline & GM Efficacy (\%) & 98.0 & 58.0 & 72.7 & 66.8 & 47.0 & 48.7 \\
\hline & $P$-value vs. placebo & $<0.0001$ & 0.0005 & $<0.0001$ & $<0.0001$ & 0.0079 & 0.0051 \\
\hline \multirow[t]{7}{*}{ Imidacloprid+permethrin } & Range & 6 to 41 & 0 to 29 & 0 to 16 & 0 to 10 & 3 to 16 & 7 to 36 \\
\hline & AM & 28.1 & 7.5 & 6.3 & 4.4 & 8.4 & 17.5 \\
\hline & AM Efficacy (\%) & 16.4 & 63.4 & 80.0 & 83.8 & 70.6 & 47.4 \\
\hline & $\mathrm{GM}^{\mathrm{c}}$ & $24.6^{a}$ & $3.5^{\mathrm{b}}$ & $4.2^{\mathrm{b}}$ & $3.3^{c}$ & $7.2^{\mathrm{b}}$ & $14.7^{b}$ \\
\hline & GM Efficacy (\%) & 23.7 & 80.8 & 85.9 & 87.0 & 73.8 & 51.9 \\
\hline & $P$-value vs. placebo & 0.3990 & $<0.0001$ & $<0.0001$ & $<0.0001$ & 0.0001 & 0.0259 \\
\hline & $P$-value vs. sarolaner & $<0.0001$ & 0.0578 & 0.1025 & 0.0231 & 0.0630 & 0.8596 \\
\hline
\end{tabular}

${ }^{c}$ Geometric means within columns with the same superscript are not significantly different $(P>0.05)$

Table 3 The Range, Arithmetic (AM) and Geometric (GM) Mean live Dermacentor reticulatus counts, and efficacy relative to placebo at 24 hours after treatment and re-infestations for dogs treated with either a single oral dose of sarolaner or a single topical application of imidacloprid+permethrin on Day 0

\begin{tabular}{|c|c|c|c|c|c|c|c|}
\hline \multirow[t]{2}{*}{ Treatment } & & \multicolumn{6}{|c|}{ Day of treatment or re-infestation } \\
\hline & & 0 & 7 & 14 & 21 & 28 & 35 \\
\hline \multirow[t]{3}{*}{ Placebo } & Range & 21 to 40 & 10 to 37 & 15 to 37 & 15 to 38 & 18 to 33 & 7 to 41 \\
\hline & AM & 30.1 & 25.0 & 28.4 & 28.1 & 28.0 & 27.0 \\
\hline & $\mathrm{GM}^{\mathrm{d}}$ & $28.7^{\mathrm{a}}$ & $23.0^{\mathrm{a}}$ & $26.6^{\mathrm{a}}$ & $26.7^{\mathrm{a}}$ & $26.9^{\mathrm{a}}$ & $24.0^{\mathrm{a}}$ \\
\hline \multirow[t]{6}{*}{ Sarolaner } & Range & 0 to 0 & 0 to 1 & 0 to 0 & 0 to 1 & 0 to 3 & 0 to 5 \\
\hline & AM & 0.0 & 0.3 & 0.0 & 0.1 & 0.9 & 1.1 \\
\hline & AM Efficacy (\%) & 100.0 & 99.0 & 100.0 & 99.6 & 96.9 & 95.8 \\
\hline & $G M^{d}$ & $0.0^{c}$ & $0.2^{c}$ & $0.0^{c}$ & $0.1^{c}$ & $0.5^{c}$ & $0.6^{c}$ \\
\hline & GM Efficacy (\%) & 100 & 99.3 & 100 & 99.8 & 98.0 & 97.6 \\
\hline & $P$-value vs. placebo & $<0.0001$ & $<0.0001$ & $<0.0001$ & $<0.0001$ & $<0.0001$ & $<0.0001$ \\
\hline \multirow[t]{7}{*}{ Imidacloprid+permethrin } & Range & 7 to 33 & 0 to 8 & 0 to 18 & 0 to 11 & 0 to 17 & 1 to 32 \\
\hline & AM & 15.6 & 2.3 & 4.6 & 3.3 & 6.3 & 14.5 \\
\hline & AM Efficacy (\%) & 48.1 & 91.0 & 83.7 & 88.4 & 77.7 & 46.3 \\
\hline & $G M^{d}$ & $13.9^{b}$ & $1.3^{b}$ & $2.3^{b}$ & $1.9^{\mathrm{b}}$ & $4.1^{b}$ & $10.4^{b}$ \\
\hline & GM Efficacy (\%) & 51.5 & 94.3 & 91.3 & 93.0 & 84.9 & 56.8 \\
\hline & $P$-value vs. placebo & 0.0282 & $<0.0001$ & $<0.0001$ & $<0.0001$ & $<0.0001$ & 0.0124 \\
\hline & $P$-value vs. sarolaner & $<0.0001$ & 0.0451 & 0.0005 & 0.0045 & 0.0006 & $<0.0001$ \\
\hline
\end{tabular}

\footnotetext{
${ }^{\mathrm{d}}$ Geometric means within columns with the same superscript are not significantly different $(P>0.05)$
} 


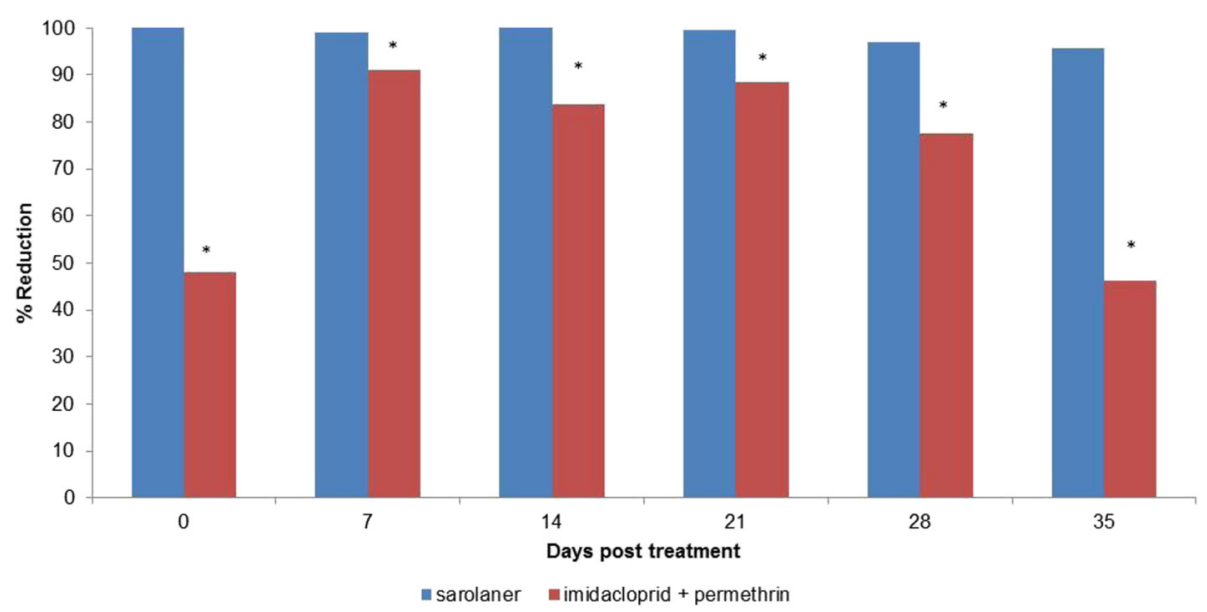

Fig. 1 Percent efficacy based on arithmetic mean Dermacentor reticulatus counts relative to placebo at 24 hours after treatment and weekly post-treatment re-infestation for dogs treated with either a single oral dose of sarolaner or a single topical application of imidacloprid plus permethrin on Day 0

Forimidacloprid + permethrin, the reduction in AM (GM) mean tick counts ranged from $44.9 \%(50.2 \%)$ to $75.8 \%$ (82.0\%), and were significantly lower $(P \leq 0.0331)$ than placebo on all days. GM live tick counts for the two products were significantly different from each other on Days 0,7 and $21(P \leq 0.0077)$.

Twelve hours after treatment the reduction in AM (GM) tick counts was $95.9 \%$ (98.0\%) for sarolaner and $16.4 \%(23.7 \%)$ for imidacloprid + permethrin. GM live tick counts for sarolaner were significantly lower $(P<0.0001)$ than for placebo, but were not for imidacloprid + permethrin $(P=0.3990)$ versus placebo. On the sarolaner-treated dogs only up to 7 live ticks were found, while on the imidacloprid + permethrin treated dogs up to 41 live ticks were recovered. Twelve hours after weekly re-infestation the reduction in AM (GM) tick counts for sarolaner ranged from $45.2 \%$ $(47.0 \%)$ to $72.4 \%(72.7 \%)(P \leq 0.0079$ on all days vs placebo). Forimidacloprid + permethrin, the reduction in AM (GM) mean tick counts ranged from $47.4 \%$ (51.9 \%) to $83.8 \%(87.0 \%)(P \leq 0.0259$ on all days vs. placebo). GM live tick counts for sarolaner were significantly lower than those for imidacloprid + permethrinon Days 0 and 21 $(P \leq 0.0231)$.

Twenty four hours after treatment no live ticks were found on any sarolaner-treated dog (100\% efficacy), while up to 33 live ticks were recovered from imidacloprid + permethrin treated dogs, representing $48.1 \%$ (51.5\%) efficacy. GM live tick counts were significantly lower than placebo for sarolaner $(P<0.0001)$ and imidacloprid + permethrin $(P \leq 0.0282)$. Twenty four hours after weekly re-infestation, the reduction in AM (GM) tick counts for sarolaner was above $95.8 \%$ (97.6\%) until Day $35(P<0.0001$ vs. placebo on all days). Forimidacloprid + permethrin, the percentage reduction in AM (GM) tick counts ranged from $91.0 \%$ (94.3\%), to $46.3 \%$ (56.8\%) ( $P \leq 0.0124$ vs. placebo on all days). Geometric mean live tick counts for sarolaner were significantly lower than those for imidacloprid + permethrin on all days $(P \leq 0.0451)$. There were no sarolaner-related adverse reactions during the study. One imidacloprid + permethrin-treated dog developed erythema on Day 0 at the site of treatment application.

\section{Discussion}

A rapid speed of kill prevents the direct adverse effects of tick attachment and feeding, and reduces the risk for transmission of tick-borne pathogens. In this study, a single dose of sarolaner significantly reduced $D$. reticulatus tick counts within 8 hours after treatment, and within 12 hours after re-infestation for 35 days. Efficacy above $90 \%$ was achieved within 24 hours and maintained for 35 days after treatment. The persistent and rapid speed of kill against $D$. reticulatus for 5 weeks is consistent with the rapid efficacy of sarolaner within 24 hours against Ixodes ricinus, Ixodes scapularis and Amblyomma maculatum for at least 4 weeks [11].

The efficacy of imidacloprid + permethrin within 48 hours of treatment against $D$. reticulatus in dogs has previously been described, with adequate efficacy in the first two weeks after treatment and a decline in efficacy from the third week onwards $[15,16]$. Additionally, the efficacy of imidacloprid + permethrin at 24 hours posttreatment or re-infestation was below $90 \%$ efficacy throughout the entire month in another study [17]. Similarly, in the present study, based on arithmetic mean the efficacy of imidacloprid + permethrin at 24 hours was below $90 \%$ at all time-points except on Day 7 . As a result, significantly $(P \leq 0.0451)$ more ticks were found 
at 24 hours on imidacloprid + permethrin-treated dogs compared to sarolaner-treated dogs throughout the study. The fact that imidacloprid + permethrin had no significant effect within 12 hours after treatment and $<50 \%$ efficacy at 24 hours, as well as the lower efficacy throughout the month at all time-points except at 24 hours on Day 7, are evidence of a slow speed of kill for imidacloprid + permethrin.

The ornate dog tick is a well-known vector of $B$. canis. Transmission of babesiosis is considered to occur within 24 to 72 hours of tick attachment $[13,14]$. As dogs can be exposed to infected ticks throughout the treatment interval, both persistent efficacy and rapid speed of kill are required to decrease the risk of babesiosis. Under field conditions imidacloprid + permethrin has demonstrated a $94.4 \%$ reduction in the incidence of infection with Babesia spp in dogs [18]. Therefore the faster and more persistent killing effect of sarolaner against $D$. reticulatus for at least 5 weeks in the present study highlights the potential of sarolaner to reduce the transmission of babesiosis to dogs.

\section{Conclusions}

This study confirmed the rapid and consistent acaricidal efficacy of sarolaner against $D$. reticulatus after a single oral administration and demonstrated that ticks were killed rapidly with $>90 \%$ efficacy within 24 hours for at least 5 weeks. Efficacy of Simparica was higher at 24 hours compared to Advantix throughout the study.

\section{Competing interests}

The study reported here was funded by Zoetis, Florham Park, NJ. CB, TG, OC, SPM, and RHS are current employees of Zoetis. HE was an independent investigator contracted for the study. There were no conflicting interests that could have influenced the conduct and reporting of this study.

\section{Authors' contributions}

Authors assisted with the study design, study conduct, interpretation of the data and manuscript writing. All authors read and approved the final version of the manuscript.

\section{Acknowledgements}

The authors would like to thank Douglas Rugg for his assistance in drafting the manuscript.

\section{Author details}

'Zoetis, Veterinary Medicine Research and Development, Mercuriusstraat 20, Zaventem B-1930, Belgium. ${ }^{2}$ ClinVet International (pty) Ltd, Uitsigweg, Bainsvlei 9338, Bloemfontein, Republic of South Africa. ${ }^{3}$ Zoetis, Veterinary Medicine Research and Development, 333 Portage St., Kalamazoo, MI 49007, USA.

Received: 18 December 2015 Accepted: 4 February 2016

Published online: 24 February 2016

\section{References}

1. Beck S, Schreiber C, Schein E, Krücken J, Baldermann C, Pachnicke S, von Samson-Himmelstjerna G, Kohn B. Tick infestation and prophylaxis of dogs in northeastern Germany: a prospective study. Ticks Tick Borne Dis. 2014;5: 336-42.

2. Claerebout E, Losson B, Cochez C, Casaert S, Dalemans AC, De Cat A Madder M, Saegerman C, Heyman P, Lempereur L. Ticks and associated pathogens collected from dogs and cats in Belgium. Parasit Vectors. 2013; 6:183.

3. Zygner W, Górski P, Wedrychowicz H. New localities of Dermacentor reticulatus tick (vector of Babesia canis canis) in central and eastern Poland. Pol J Vet Sci. 2009;12:549-55.

4. Beugnet F, Marié JL. Emerging arthropod-borne diseases of companion animals in Europe. Vet Parasitol. 2009;163:298-305.

5. Földvári G, Farkas R. Ixodid tick species attaching to dogs in Hungary. Vet Parasitol. 2005:129:125-31.

6. Siroký P, Kubelová M, Bednář M, Modrý D, Hubálek Z, Tkadlec E. The distribution and spreading pattern of Dermacentor reticulatus over its threshold area in the Czech Republic-how much is range of this vector expanding? Vet Parasitol. 2011;183:130-5.

7. Dautel H, Dippel C, Oehme R, Hartelt K, Schettler E. Evidence for an increased geographical distribution of Dermacentor reticulatus in Germany and detection of Rickettsia sp. RpA4. Int J Med Microbiol. 2006;296 Suppl 40:149-56.

8. Dantas-Torres F, Chomel BB, Otranto D. Ticks and tick-borne diseases: a One Health perspective. Trends Parasitol. 2012;28:437-46.

9. Matijatko V, Torti M, Schetters TP. Canine babesiosis in Europe: how many diseases? Trends Parasitol. 2012;28:99-105.

10. Geurden T, Becskei C, Grace S, Strube C, Doherty P, Liebenberg J, Mahabir SP, Slootmans N, Lloyd A, Six RH. Efficacy of a novel oral formulation of sarolaner (Simparica ${ }^{\mathrm{TM}}$ ) against four common tick species infesting dogs in Europe. Vet Parasitol, submitted

11. Six RH, Geurden T, Carter L, Everett WR, McLoughlin A, Mahabir SP, Myers MR, Slootmans N. Knock-down and speed of kill of sarolaner (Simparica ${ }^{\text {TM }}$ ) against induced infestations of three species of ticks (Amblyomma maculatum, Ixodes scapularis, Ixodes ricinus). Vet Parasitol, submitted

12. Marchiondo AA, Holdsworth PA, Fourie LJ, Rugg D, Hellmann K, Snyder DE, et al. World Association for the Advancement of Veterinary Parasitology (W. A.A.V.P.) 2nd. ed.: Guidelines for evaluating the efficacy of parasiticides for the treatment, prevention and control of flea and tick infestations on dogs and cats. Vet Parasitol. 2013;194:84-97.

13. Little SE. Changing paradigms in understanding transmission of canine tickborne diseases: the role of interrupted feeding and intrastadial transmission. In: 2nd Canine Vector-Borne Disease (CVBD) Symposium. Mezara del Vallo, Sicily, Italy. 2007; pp. 30-34.

14. Martinod S, Brossard M, Moreau Y. Immunity of Dogs against Babesia canis, Its Vector Tick Dermacentor reticulatus, and Ixodes ricinus in Endemic Area. J Parasitol. 1985;71:269-73.

15. Tielemans E, Manavella C, Pollmeier M, Chester T, Murphy M, Gale B. Comparative acaricidal efficacy of the topically applied combinations fipronil/(S)-methoprene, permethrin/imidacloprid and metaflumizone/ amitraz against Dermacentor reticulatus, the European dog tick (ornate dog tick, Fabricius, 1794) in dogs. Parasite. 2010;17:343-8.

16. Doyle V, Beugnet F, Carithers D. Comparative efficacy of the combination fipronil-(S)-methoprene and the combination permethrin-imidacloprid against Dermacentor reticulatus, the European dog tick, applied topically to dogs. Vet Ther Winter. 2005;6:303-10.

17. Fourie JJ, Beugnet F, Ollagnier C, Pollmeier MG. Study of the sustained speed of kill of the combination of fipronil/amitraz/(S)-methoprene and the combination of imidacloprid/permethrin against Dermacentor reticulatus, the European dog tick. Parasite. 2011;18:319-23.

18. Otranto D, De Caprariis D, Lia RP, Tarallo V, Lorusso V, Testini G, Stanneck D. Prevention of endemic canine vector-borne diseases using imidacloprid $10 \%$ and permethrin $50 \%$ in young dogs: a longitudinal field study. Vet Parasitol. 2010;172:323-32. 